\title{
The Nullity of Compact Minimal Real Hypersurfaces in a Complex Projective Space
}

\author{
Tohru GOTOH
}

\author{
Chiba University \\ (Communicated by T. Nagano)
}

Dedicated to Professor Tsunero Takahashi on his 60th birthday

\section{Introduction.}

Let $M$ be a compact minimal submanifold of a Riemannian manifold $\tilde{M}$. Then the general situation of the problem we will investigate in this paper is the following:

Problem 1.1. Estimate the index or the nullity of $M$ from below, and furthermore determine $M$ whose index or nullity attains the lowest bound in the estimation.

In general, this problem is very difficult, so it seems to be interesting and important to observe for specific ambient manifolds. Concerning with this problem, the following two results are well-known:

EXAMPLE 1.2 (J. Simons [S]). In the case where $\tilde{M}$ is the $n$-dimensional sphere and dimension of $M$ is $p$, then the inequalities ind $(M) \geq n-p$ and $\operatorname{nul}(M) \geq(p+1)(n-p)$ hold. Moreover, in each inequality, the equality holds only when $M$ is totally geodesic.

EXAMPLE 1.3 (Y. Kimura $[\mathrm{K}]$ ). In the case where $\tilde{M}$ is the complex projective space with complex dimension $n$ and $M$ a Kähler submanifold with complex dimension $p$, then inequality $\operatorname{nul}(M) \geq 2(p+1)(n-p)$ holds and equality holds only when $M$ is totally geodesic.

Here ind $(M)$ and $\operatorname{nul}(M)$ stand for the index and the nullity of $M$ respectively. Moreover Ohnita [O, section 6] has obtained a generalization of their results above to the case of compact rank-1 symmetric spaces.

The purpose of this paper is to investigate the problem (1.1), mainly for the nullity, in the case where $\tilde{M}$ is the $n$-dimensional complex projective space $P^{n} C$ with the Fubini-Study metric of constant holomorphic sectional curvature 4, and $M$ a compact oriented minimal real hypersurface in $P^{n} C$. It should be noted that in the examples above the minimal submanifold with minimum index or nullity is always totally 
geodesic. On the contrary, it is known that there exsists no totally geodesic, in fact, no totally umbilic real hypersurface in $P^{n} C$. This is a result of a simple application of the equation of Codazzi. However there is a 'nice' family of minimal real hypersurfaces $M_{p, q}^{c}$, often called the model hypersurfaces, which are defined as follows ([L]):

We denote by $S^{d}(r)$ an Euclidean $d$-sphere with radius $r$. For each non-negative integers $p$ and $q$ with $p+q=n-1$, we put $r_{p}=\sqrt{(2 p+1) / 2 n}$ and $r_{q}=\sqrt{(2 q+1) / 2 n}$. Then the Riemannian product $S^{2 p+1}\left(r_{p}\right) \times S^{2 q+1}\left(r_{q}\right)$ is naturally imbedded minimally in $S^{2 n+1}(1)$. A real hypersurface $M_{p, q}^{c}$ is defined by the commutative diagram

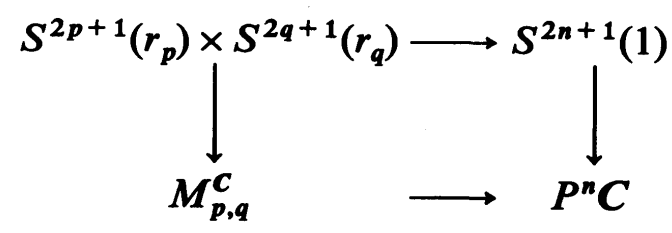

where $S^{1}(1) \rightarrow S^{2 n+1}(1) \rightarrow P^{n} C$ is the Hopf fibration. Especially $M_{0, n-1}^{C}$ is called geodesic hypersphere, which has more fundamental properties among the family $M_{p, q}^{c}$. Many properties which distinguish the model hypersurfaces in the class of real hypersurfaces in $P^{n} C$ have been known.

Now we state the main theorem which asserts that the value of the nullity distinguishes the geodesic hypersphere $M_{0, n-1}^{c}$ from other minimal real hypersurfaces in $P^{n} C$ :

MAIN THEOREM. Let $M$ be a compact oriented minimal real hypersurface in $P^{n} C$. Then an inequality nul $(M) \geq 2 n$ holds. Moreover, in the inequality, the equality holds if and only if $M$ is the geodesic hypersphere $M_{0, n-1}^{c}$.

This theorem will be proved in section 4 (Proposition 4.7) and section 5 (Theorem 5.6). In section 6, we will refer to some remarks on index part of problem (1.1).

The author would like to express his sincere thanks to Prof. H. Urakawa and Prof. Y. Ohnita for their kind advices, and to Prof. R. Takagi for encouragement.

\section{Jacobi operator.}

Let $M$ be a compact oriented minimal submanifold (without boundary) immersed in a Riemannian manifold $\tilde{M}$. We denote by $T M$ and $N M$ the tangent and the normal bundle of $M$ respectively and ${ }^{\perp}: T \tilde{M} \rightarrow N M$ the orthogonal projection. Let $\nabla^{M}$ and $\nabla^{M}$ be the Riemannian connection of $M$ and $\tilde{M}$ respectively. Then a metric connection $\nabla^{\perp}$ on $N M$, called the normal connection, is defined as $\nabla_{X}^{\perp} V=\left(\nabla_{X}^{\mathscr{T}} V\right)^{\perp}$ for $X \in \Gamma(T M)$ and $V \in \Gamma(N M)$. Here $\Gamma(\cdot)$ indicates the space of all $C^{\infty}$-sections of the bundle. we set

Now the Jacobi operator $\mathfrak{I}_{M}$ of $M$ is defined as follows ([S]): For each $V \in \Gamma(N, M)$,

$$
\mathfrak{I}_{M} V=-\sum\left(\nabla_{e_{j}}^{\perp} \nabla_{e_{j}}^{\perp}-\nabla_{\nabla_{j_{j}}}^{\perp}\right) V+\sum\left(R^{\mathscr{A}}\left(e_{j}, V\right) e_{j}\right)^{\perp}-\sum B\left(A_{V} e_{j}, e_{j}\right)
$$


where $\left\{e_{j}\right\}$ is an orthonormal local frame field of $M, R^{\tilde{M}}$ is the curvature tensor of $\tilde{M}$, $B$ is the second fundamental form of the immersion, and $A_{V}$ is the shape operator in the direction $V$. Then if $M_{t}$ is a variation of $M=M_{0}$ with variational vector field $V \in \Gamma(N M)$, the second variational formula of the volume functional is given by

$$
\left.\frac{d^{2}}{d t^{2}}\right|_{t=0} \operatorname{Vol}\left(M_{t}\right)=\int_{M}\left\langle I_{M} V, V\right\rangle d v,
$$

where $\langle\cdot, \cdot\rangle$ is the induced fibre metric on $N M$ and $d v$ denotes the volume form of $M$. It is known that $\mathfrak{I}_{M}$ is a self-adjoint strongly elliptic partial differential operator acting on $\Gamma(N M)$, and has discrete eigenvalues $\mu_{1}<\mu_{2}<\cdots \rightarrow \infty$. If we denote by $E_{\mu}\left(\mathfrak{I}_{M}\right)$ the $\mu$-eigenspace of $\mathfrak{I}_{M}$, the index and the nullity of $M$ are given by

$$
\operatorname{ind}(M)=\sum_{\mu<0} \operatorname{dim} E_{\mu}\left(\mathfrak{I}_{M}\right) \quad \text { and } \quad \operatorname{nul}(M)=\operatorname{dim} E_{0}\left(\mathfrak{I}_{M}\right)
$$

respectively.

From now on, we consider the case where $\tilde{M}$ is $P^{n} C$ and $M$ is a compact oriented minimal real hypersurface. In this case $N M$ is a trivial line bundle. Throughout of this paper, $v$ denotes a globally defined unit normal field and $A$ the shape operator in the direction $v$. Because the space $C^{\infty}(M)$ of all the smooth functions on $M$ is isomorphic to $\Gamma(N M)$ by corresponding $f \in C^{\infty}(M)$ to $f v \in \Gamma(N M)$, we regard $\mathfrak{I}_{M}$ as an operator acting on $C^{\infty}(M)$. We note the curvature tensor $R^{P}$ of $P^{n} C$ is given as

$$
\begin{aligned}
R^{P}(X, Y) Z= & g(Y, Z) X-g(X, Z) Y+g(J Y, Z) J X-g(J X, Z) J Y \\
& +2 g(X, J Y) J Z, \quad X, Y, Z \in T P^{n} C,
\end{aligned}
$$

where $g$ and $J$ denote the metric tensor and the complex structure of $P^{n} C$ respectively. Therefore, from (2.1) and (2.2), we obtain

$$
\mathfrak{I}_{M} f=\Delta_{M} f-\left(2(n+1)+\|A\|^{2}\right) f, \quad f \in C^{\infty}(M),
$$

where $\Delta_{M}$ denotes the (non-negative) Laplacian of $M$ acting on $C^{\infty}(M)$ and $\|A\|^{2}$ denotes the square of the length of $A$.

In the case where $M$ is $M_{p, q}^{c},\|A\|^{2}=2(n-1)$. Therefore (2.3) implies

$$
\mathfrak{I}_{M_{p, q}^{c}} f=\Delta_{M_{p, q}^{c}} f-4 n f, \quad f \in C^{\infty}\left(M_{p, q}^{c}\right) \text {. }
$$

In paticular, the index and the nullity of $M_{p, q}^{c}$ are computed from its spectrum.

ReMARK 2.5. It should be mentioned that (2.3) implies the instability of any compact oriented minimal real hypersurface $M$ in $P^{n} C$, namely ind $(M)>0$.

\section{The spectrum, the index, and the nullity of $M_{p, q}^{c}$.}

In this section we compute the index and the nullity of the model hypersurfaces 
$M_{p, q}^{c}$. For a Riemannian manifold $M$, we denote by $\operatorname{Spec}(M)$ the set of all eigenvalues of $\Delta_{M}$. By using the diagram (1.1), we can compute $\operatorname{Spec}\left(M_{p, q}^{c}\right)$ as follows (cf. [B-G-M, Chapter III]). We consider $S^{2 p+1}\left(r_{p}\right)$ (resp. $S^{2 q+1}\left(r_{q}\right)$ ) to be contained in $C^{p+1}$ (resp. $\left.C^{q+1}\right)$ and denote by $\left(z_{1}, \cdots, z_{p+1}\right)$ (resp. $\left(w_{1}, \cdots, w_{q+1}\right)$ ) the canonical complex coordinate of $C^{p+1}\left(\right.$ resp. $\left.C^{q+1}\right)$. For each non negative integers $s$ and $t$, let $\mathscr{P}_{s, t}^{2 p+1}(z, \bar{z})$ be the space of all homogeneous polynomials with coefficients in $C$ and of bi-degree $(s, t)$ with respect to $z_{j}$ and $\bar{z}_{j}$. We put further

$$
\tilde{\mathscr{H}}_{s, t}^{2 p+1}(z, \bar{z})=\left\{P \in \tilde{\mathscr{P}}_{s, t}^{2 p+1} \mid \Delta P=0\right\}, \quad \text { where } \quad \Delta=-4 \sum \frac{\partial^{2}}{\partial z_{j} \partial \bar{z}_{j}} .
$$

The spaces $\tilde{\mathscr{P}}_{u, v}^{2 q+1}(w, \bar{w})$ and $\tilde{\mathscr{H}}_{u, v}^{2 q+1}(w, \bar{w})$ are defined similarly. The circle group $S^{1}=\left\{e^{\sqrt{-1} \theta} \mid \theta \in R\right\}$ acts naturally on the tensor product $\mathscr{\mathscr { P }}_{s, t}^{2 p+1}(z, \bar{z}) \otimes \mathscr{\mathscr { P }}_{u, v}^{2 q+1}(w, \bar{w})$. Then we have easily

LeMMA 3.1. For each $P \in \mathscr{P}_{s, t}^{2 p+1}(z, \bar{z})$ and $Q \in \mathscr{P}_{u, v}^{2 q+1}(w, \bar{w}), \quad P Q \in$ $\tilde{\mathscr{P}}_{s, t}^{2 p+1}(z, \bar{z}) \otimes \widetilde{\mathscr{P}}_{u, v}^{2 q+1}(w, \bar{w})$ is invariant by $S^{1}$ if and only if $s-t+u-v=0$.

It is known

$$
\operatorname{Spec}\left(S^{2 p+1}\left(r_{p}\right)\right)=\left\{\lambda_{k}^{2 p+1}=\frac{2 n k(k+2 p)}{2 p+1} \mid k=0,1, \cdots\right\}
$$

and

$$
\operatorname{Spec}\left(S^{2 q+1}\left(r_{q}\right)\right)=\left\{\lambda_{l}^{2 q+1}=\frac{2 n l(l+2 q)}{2 q+1} \mid l=0,1, \cdots\right\}
$$

so that

$$
\operatorname{Spec}\left(S^{2 p+1}\left(r_{p}\right) \times S^{2 q+1}\left(r_{q}\right)\right)=\left\{\lambda_{k}^{2 p+1}+\lambda_{l}^{2 q+1} \mid k, l=0,1, \cdots\right\} .
$$

Moreover the eigenspace (precisely its complexification) of eigenvalue $\lambda_{k}^{2 p+1}+\lambda_{l}^{2 q+1}$ is given by $\tilde{\mathscr{H}}_{s, t}^{2 p+1}(z, \bar{z}) \otimes \tilde{\mathscr{H}}_{u, v}^{2 q+1}(w, \bar{w})$. Because $S^{2 p+1}\left(r_{p}\right) \times S^{2 q+1}\left(r_{q}\right)$ is a principal $S^{1}$-bundle over $M_{p, q}^{c}$, eigenfunctions of $M_{p, q}^{c}$ correspond to eigenfunctions of $S^{2 p+1}\left(r_{p}\right) \times S^{2 q+1}\left(r_{q}\right)$ which are invariant by $S^{1}$. Therefore for $\lambda \in \operatorname{Spec}\left(M_{p, q}^{c}\right)$, its multiplicity, denoted by $\mathrm{m}(\lambda)$, is given as follows:

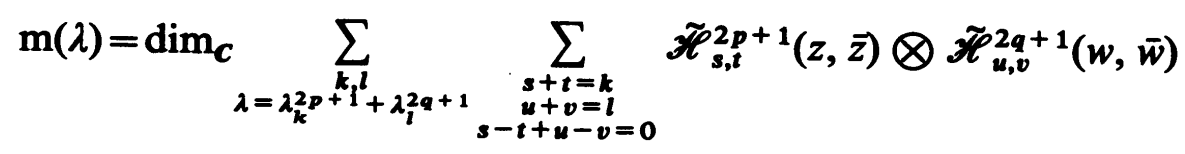

$$
\begin{aligned}
& =\sum \sum\left\{\left(\begin{array}{c}
p+s \\
s
\end{array}\right)\left(\begin{array}{c}
p+t \\
t
\end{array}\right)-\left(\begin{array}{c}
p+s-1 \\
s-1
\end{array}\right)\left(\begin{array}{c}
p+t-1 \\
t-1
\end{array}\right)\right\} \\
& \times\left\{\left(\begin{array}{c}
q+u \\
u
\end{array}\right)\left(\begin{array}{c}
q+v \\
v
\end{array}\right)-\left(\begin{array}{c}
q+u-1 \\
u-1
\end{array}\right)\left(\begin{array}{c}
q+v-1 \\
v-1
\end{array}\right)\right\} .
\end{aligned}
$$


From (2.3) and (3.2), we obtain

$$
\left\{\begin{array}{l}
\operatorname{nul}\left(M_{p, q}^{c}\right)=\mathrm{m}(4 n)=2(p+1)(q+1), \quad \text { in particular } \operatorname{nul}\left(M_{0, n-1}^{c}\right)=2 n, \\
\operatorname{ind}\left(M_{p, q}^{c}\right)=\sum_{\lambda<4 n} \mathrm{~m}(\lambda)=1
\end{array}\right.
$$

\section{The estimation of the nullity.}

This section is devoted to a proof of the estimation part of the theorem. The metric tensors of $P^{n} C$ and $M$ are denoted by the same letter $g$, while the Riemannian connections of them are denoted by $\nabla^{P}$ and $\nabla^{M}$ respectively.

For a Killing vector field $Z$, we define a function $f_{Z}$ on $M$ by $f_{Z}=g(Z, v)$, and set

$$
C_{\mathcal{A}}^{\infty}(M)=\left\{f_{Z} \in C^{\infty}(M) \mid Z \text { is a Killing vector field on } P^{n} C\right\} \text {. }
$$

Firstly, although the following lemma is due to [S, Corollary 3.3.1], we give its proof for the sake of completeness.

LEMMA 4.1. $\mathfrak{I}_{M}\left(C_{\Re}^{\infty}(M)\right)=\{0\}$.

ProOF. Let $e_{1}, \cdots, e_{2 n-1}$ be an orthonormal frame field about a point, say $x$, in $M$ such that $\nabla^{M} e_{j}=0$ at $x$ for $j=1, \cdots, 2 n-1$. Then we have

$$
\Delta_{M} f_{\mathrm{Z}}=-\sum g\left(\nabla_{e_{j}}^{P_{e_{j}}} \nabla_{e^{P}} Z, v\right)-2 \sum g\left(\nabla_{e_{j}}^{P} Z, \nabla_{e_{j}}^{P} v\right)-\sum g\left(Z, \nabla_{e_{j}}^{P_{e_{j}}} \nabla_{e_{j}}^{P} v\right) \text {. }
$$

Now since $Z$ is a Killing vector field on $P^{n} C$ and $A$ is symmetric,

$$
\sum g\left(\nabla_{e_{j}}^{P} Z, \nabla_{e_{j}}^{P} v\right)=-g\left(\nabla_{e_{j}}^{P} Z, A e_{j}\right)=0
$$

Similarly we obtain

$$
\begin{aligned}
-\sum g\left(\nabla_{e_{j}}^{P} \nabla_{e_{j}}^{P} Z, v\right) & =\sum g\left(R^{P}\left(e_{j}, v\right) Z, e_{j}\right) \\
& =2(n+1) f_{Z},
\end{aligned}
$$

where the last equation comes from (2.2).

On the other hand, the minimality of $M$ implies $\sum\left(\nabla_{e_{j}}^{M} A\right) e_{j}=0$. Thus

$$
\begin{aligned}
-\sum g\left(Z, \nabla_{e_{j}}^{P} \nabla_{e_{j}}^{P} v\right) & =\sum g\left(Z,\left(\nabla_{e_{j}}^{M} A\right) e_{j}\right)+\|A\|^{2} f_{\mathrm{Z}} \\
& =\|A\|^{2} f_{Z} .
\end{aligned}
$$

Therefore (2.3), (4.2), (4.3), (4.4) and (4.5) imply

$$
\mathfrak{I}_{M} f_{Z}=\Delta_{M} f_{Z}-\left\{2(n+1)+\|A\|^{2}\right\} f_{Z}=0 \text {. }
$$

Secondly, we note the following lemma which holds whether $M$ is minimal or not.

LEMMA 4.6. For each point $x \in M$ and for each pair $(v, \omega) \in T_{x} P^{n} C \oplus$ $\operatorname{Hom}\left(T_{x} M, N_{x} M\right)$, there exists a Killing vector field $Z$ on $P^{n} C$ such that $Z_{x}=v$ and $\left(\nabla^{\perp} Z^{\perp}\right)_{x}=\omega$. 
Now from lemma (4.1) and (4.6), we have the following estimation for the nullity:

Proposition 4.7. For a point $x$ in $M$, define a mapping

$$
\Phi_{x}: C_{\Re}^{\infty}(M) \rightarrow N_{x} M \oplus \operatorname{Hom}\left(T_{x} M, N_{x} M\right)
$$

by

$$
\Phi_{x}\left(f_{Z}\right)=\left(f_{Z}(x) v_{x},\left(d f_{Z}\right)_{x} \otimes v_{x}\right)=\left(Z_{x}^{\perp},\left(\nabla^{\perp} Z^{\perp}\right)_{x}\right) .
$$

Then $\Phi_{x}$ is a surjective linear mapping. In particular

$$
\operatorname{nul}(M) \geq \operatorname{dim} C_{\Re}^{\infty}(M) \geq 2 n .
$$

\section{A minimal real hypersurface with nullity $2 \boldsymbol{n}$.}

As we have shown, the nullity of the geodesic hypersphere $M_{0, n-1}^{c}$ attains the lowest bound of the estimation obtained in proposition 4.7, namely $\operatorname{nul}\left(M_{0, n-1}^{c}\right)=2 n$. In this section, we will prove that the geodesic hypersphere is the only compact minimal real hypersurface in $\dot{P}^{n} C$ with nullity $2 n$.

First of all, we prove

Proposition 5.1. If $\operatorname{nul}(M)=2 n$, then $M$ must be homogeneous. Here homogeneity means $M$ is an orbit of an analytic subgroup of the isometry group of $P^{n} C$.

Proof. For each $x \in M$ and each $X \in T_{x} M$ we show the existence of a Killing vector field $Z$ on $P^{n} C$ which is tangent to $M$ everywhere on $M$ and $Z_{x}=X$. have

In fact, taking a Killing vector field $Z$ on $P^{n} C$ such as $Z_{x}=X$ and $\left(\nabla^{\perp} Z^{\perp}\right)_{x}=0$, we

$$
\Phi_{x}\left(f_{z}\right)=0 \text {. }
$$

If $\operatorname{nul}(M)=2 n$, the mapping $\Phi_{x}$ must be an isomorphism. Therefore (5.2) implies $f_{Z}=0$, so that $Z$ is tangent to $M$ everywhere on $M$.

Now we let $P^{n} C=U(n+1) / U(1) \times U(n)=G / H$ and $\pi: U(n+1) \rightarrow P^{n} C$ the natural projection. Putting

$$
\mathfrak{p}=\left\{\left(\begin{array}{cc}
0 & -{ }^{t \bar{\zeta}} \\
\zeta & 0
\end{array}\right) \in \mathfrak{g} \mid \zeta \in C^{n}\right\},
$$

then we have the orthogonal decomposition

$$
\mathfrak{g}=\mathfrak{h}+\mathfrak{p},
$$

where $g$ and $\mathfrak{h}$ denote the Lie algebra of $G$ and $H$ respectively. Let $o=\pi(I)$ be the origin and we identify $\mathfrak{p}$ canonically to $T_{o} P^{n} C$. Because $P^{n} C=G / H$ is isotropic, that is, the linear isotropy group acts transitively on the unit sphere in $\mathfrak{p}$, we may assume 


$$
o \in M \quad \text { and } \quad v_{o}=\left(\begin{array}{cc}
0 & -t \overline{\zeta_{0}} \\
\zeta_{0} & 0
\end{array}\right),
$$

where $\zeta_{0}={ }^{t}(1,0, \cdots, 0) \in C^{n}$. We define a vector field $\xi$ on $M$ by $\xi=-J v$ and a subspace $m$ of $T_{o} M$ to be the orthogonal complement of $\operatorname{span}\left\{\xi_{o}\right\}$. Further we define a closed subgroup $U$ of $U(n+1)$ as

$$
U=\left\{\left(\begin{array}{ccc}
1 & 0 & 0 \\
0 & 1 & 0 \\
0 & 0 & W
\end{array}\right) \in U(n+1) \mid W \in U(n-1)\right\} \cong U(n-1) .
$$

Considering $u \in U$ as an isometry of $P^{n} C$, we denote by $u_{*}$ its differential at the origin $o$. Then, as a consequence of the assumption (5.3) and definitions above, we easily obtain

LEMMA 5.4. $u(o)=o, u_{*}\left(\xi_{o}\right)=\xi_{0}$, and $u_{*}\left(v_{o}\right)=v_{o}$, and hence

$$
u_{*}\left(T_{o} M\right) \subset T_{o} M, \quad u_{*}(\mathfrak{m}) \subset \mathfrak{m} .
$$

The next lemma is a result from the condition that the nullity of $M$ is equal to $2 n$.

LeMma 5.5. Assume $\operatorname{nul}(M)=2 n$. Then every Killing vector field on $P^{n} C$ generated by $U$ is tangent to $M$ everywhere on $M$. Consequently $U$ leaves $M$ invariant, and we can consider $U$ as a subgroup of the isometry group of $M$.

Proof. Let $Z$ be an element in $\mathfrak{u}$, the Lie algebra of $U$, and $Z^{*}$ a Killing vector field generated by $Z$ :

$$
Z_{p}^{*}=\left.\frac{d(\exp t Z) p}{d t}\right|_{t=0}, \quad p \in P^{n} C .
$$

By virtue of lemma (5.4), we have

$$
\operatorname{ad}(Z) v_{0}=\left.\frac{d \operatorname{Ad}(\exp t Z) v_{0}}{d t}\right|_{t=0}=\left.\frac{d v_{0}}{d t}\right|_{t=0}=0,
$$

and hence for all $X \in \mathfrak{m}$

$$
g\left(\left(\nabla_{X}^{P} Z^{*}\right), v_{0}\right)=g\left(\operatorname{ad}(Z) X, v_{0}\right)=-g\left(X, \operatorname{ad}(Z) v_{0}\right)=0 .
$$

We also obtain $Z_{o}^{*}=0$ by lemma (5.4), and as a result

$$
\begin{aligned}
\nabla_{X}^{\perp} Z^{* \perp} & =\left(\nabla_{X}^{P} Z^{*}\right)^{\perp}-\left(\nabla_{X}^{P} Z^{* \top}\right)^{\perp} \\
& =0-g\left(A X, Z^{* \top}\right) v_{0} \\
& =0 .
\end{aligned}
$$

This implies $\Phi_{0}\left(f_{Z^{*}}\right)=0$, and on account of proposition 4.7 , we get $f_{Z^{*}}=0$ by assumption $\operatorname{nul}(M)=2 n$. 
We are now in a position to complete a proof of the following theorem: $M_{\mathbf{0}, n-1}^{C}$.

THEOREM 5.6. Assume $\operatorname{nul}(M)=2 n$, then $M$ must be the geodesic hypersphere

Proof. Since $M$ is homogeneous by lemma (5.1), $\xi$ is a principal curvature vector on $M$. Consequently the shape operator $A$ leaves $\mathfrak{m}$ invariant. Let $X, Y \in \mathfrak{m}$ with $\|X\|=\|Y\|=1$. Then there exists a certain $u \in U$ such that $u_{*} Y=X$. By lemma (5.5), this $u$ is an isometry of $M$ as well as of $P^{n} C$. So we have

$$
\begin{aligned}
g(A X, X) v_{0} & =\nabla_{X}^{P} X-\nabla_{X}^{M} X \\
& =\nabla_{u_{*} Y}^{P} u_{*} Y-\nabla_{u_{*} Y}^{M} u_{*} Y \\
& =u_{*}\left(\nabla_{Y}^{P} Y-\nabla_{Y}^{M} Y\right) \\
& =u_{*}\left(g(A Y, Y) v_{0}\right) \\
& =g(A Y, Y) v_{0} \quad \text { for all } X, Y \in m .
\end{aligned}
$$

Therefore principal curvature is unique on $\mathrm{m}$. By virtue of Takagi's classification of homogeneous real hypersurfaces in $P^{n} C([T])$, we conclude $M$ must be the geodesic hypersphere.

\section{Some remarks on index problem.}

In this last section we conclude this paper with refering a little to the index part of problem (1.1).

As was mentioned in remark (2.5), any compact oriented minimal real hypersurface $M$ satisfies an inequality

$$
\operatorname{ind}(M) \geq 1 \text {. }
$$

This estimation is sharp in the sense that model hypersurfaces $M_{p, q}^{c}$ satisfy the equality in (6.1). For this reason, we propose the following problem:

PROBLem 6.2. If the index of a compact oriented minimal real hypersurface $M$ is equal to one, is $M$ a certain model hypersurface?

Under some additional conditions, problem (6.2) can be solved affirmatively:

Proposition 6.3. Assume $M$ satisfies

$$
\lambda_{1}(M) \leq 4 n,\|A\|^{2}=\text { constant on } M, \text { and } \operatorname{ind}(M)=1,
$$

where $\lambda_{1}(M)$ denotes the first eigenvalue of $\Delta_{M}$. Then $M=M_{p, q}^{c}$ for some $p, q$.

To prove this, we need the following result:

FACT 6.4 (Lawson [L, Theorem 2]). Let $M$ be a compact minimal real hypersurface of $P^{n} C$ over which the inequality $\|A\|^{2} \leq 2(n-1)$ holds. Then $\|A\|^{2}=2(n-1)$, and 
up to isometries of $P^{n} C, M=M_{p, q}^{c}$ for some $p, q$.

Próof of Proposition 6.3. Let

$$
0=\lambda_{0}<\lambda_{1}<\cdots \rightarrow \infty
$$

be the spectrum of $\Delta_{M}$. Then since $\|A\|^{2}=$ const., the spectrum of the Jacobi operator $\mathfrak{I}_{M}$ is

$$
-2(n+1)-\|A\|^{2}<\lambda_{1}-2(n+1)-\|A\|^{2}<\cdots \rightarrow \infty .
$$

Hence the assumptions $\lambda_{1} \leq 4 n$ and $\operatorname{ind}(M)=1$ imply

$$
\|A\|^{2} \leq 2(n-1) \text {. }
$$

The assertion follows from (6.4) immediately.

REMARK 6.5. The first eigenvalues of all the model hypersurfaces equal to $4 n$, while those multiplicities differ from each other (see (3.2) and (3.3)).

REMARK 6.6. Ejiri and Ros ([R]) showed independently an estimation of the first eigenvalue of the compact CR-minimal submanifold in $P^{n} C$. Applying their estimation to a compact minimal real hypersurface $M$, we obtain

$$
\lambda_{1}(M) \leq 4 n+2-\frac{2}{2 n-1} \text {. }
$$

\section{References}

[B-G-M] M. BERGER, P. GAUduChON and E. MAZET, Le Spectre d'une Variété Riemannienne, Lecture Notes in Math. 194 (1970), Springer.

[K] Y. KIMURA, The nullity of compact Kähler submanifolds in a complex projective space, J. Math. Soc. Japan 29 (1977), 561-580.

[L] H. B. LAwson, JR., Rigidity theorem in rank-1 symmetric spaces, J. Differential Geom. 4 (1970), 349-357.

[O] Y. OHNITA, On stability of minimal submanifolds in compact symmetric spaces, Compositio Math. 64 (1987), 157-189.

[R] A. Ros, Spectral geometry of CR-minimal submanifolds in the complex projective space, Kodai Math. J. 6 (1983), 88-99.

[S] J. Simons, Minimal varieties in Riemannian manifolds, Ann. Math. 88 (1968), 62-105.

[T] R. TAKAGI, On homogeneous real hypersurfaces in a complex projective space, Osaka J. Math. 10 (1973), 495-506.

Present Address:

Department of Mathematics, College of liberal Arts, Kanazawa University, KanAZAWA, 920-11 JAPAN. 